\title{
Vegetation Interaction Game: Digital SUGOROKU of Vegetation Succession for Children
}

\author{
Akiko Deguchi ${ }^{1}$, Shigenori Inagaki ${ }^{2}$, Fusako Kusunoki ${ }^{3}$, \\ Etsuji Yamaguchi ${ }^{2}$, Yoshiaki Takeda ${ }^{2}$, and Masanori Sugimoto ${ }^{4}$ \\ ${ }^{1}$ Faculty of Education, Utsunomiya University, Japan \\ deguchia@cc.utsunomiya-u.ac.jp \\ ${ }^{2}$ Graduate School of Human Development and Environment, Kobe University, Japan \\ inagakis@kobe-u.ac.jp, etuji@opal.kobe-u.ac.jp, \\ takedayo@kobe-u.ac.jp \\ ${ }^{3}$ Faculty of Art and Design, Tama Art University \\ kusunoki@tamabi.ac.jp \\ ${ }^{4}$ Graduate School of Engineering, University of Tokyo \\ sugi@itl.t.u-tokyo.ac.jp
}

\begin{abstract}
In this study, we redesign and develop a new digital sugoroku game based on the phenomenon of vegetation succession. A practical evaluation in an elementary school that consisted of game play and fieldwork activity was conducted. The results of the evaluation showed that the game was effective in stimulating the interest of the students who participated in the game, and was able to support their learning in a joyful way.
\end{abstract}

Keywords: Digital interaction game, vegetation succession.

\section{Introduction}

Simulation games play an important role in the teaching and learning of science at school. They can support enjoyable interactive learning of complex systems in nature that are difficult to deal with in the school laboratory [1].

In our previous study, we have developed a simulation game, the "Vegetation Interaction Game" based on the vegetation succession, which is a digital sugoroku board game that simulates the real forest area of Mt. Rokko in Japan. In this sugoroku board game, a piece corresponds to a plant, and a grid of sugoroku to the relative succession phase around the plants. In playing the game, each child handles one piece. This condition supports children's playing the role of agent in the simulation. Moreover, we conducted an experimental evaluation in a university in Japan. The results showed the game was effective in stimulating the interest of students, and in supporting their learning in a joyful way[2].

In this study, we made some improvements in game interface to strengthen the enjoyability and understandability of the game. Additionally, we conducted a practical evaluation that consisted of two phases; game and fieldwork activity phase. This issue shows the development and improvement of our "Vegetation Interaction Game," and the results of practical evaluation. 


\section{Development and Improvement of the Game}

Figure 1 is the main window of the digital game; it shows the face of the sugoroku board. 6 plant pieces represent 6 characteristic plants that grow in the Mt. Rokko region. The size of the sugoroku board was set at $1024 * 768$ pixels. The surrounding part is the grid area of

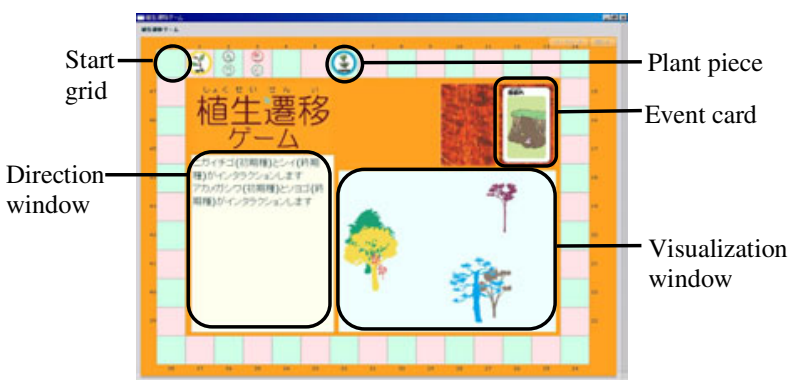

Fig. 1. Main window of sugoroku board the sugoroku board. The central part houses the event cards area, a direction window to move pieces, and a visualization window to show vegetation succession according to the progress of the game.

6 players can participate in one game. Each player handles one piece. Players draw event cards, one at a time, by clicking in turn. When a plant piece advances ahead on the board grids, it implies that the plant is dominant in that particular environment. Each piece is to be moved by the number of grids that the current event card indicates. These directions are shown in the central part of the main window. If more than one piece takes the same position on the grid, they will be moved along the grids as indicated by the rules governing the interaction between plants. A game finishes when all event cards are drawn.

In this study, following points were improved based on the feedback of the previous experimental evaluation; (a) layout of the interface to make it more understandable, (b) illustration of event cards and visualization window to make them more scientifically valid and enjoyable for children.

\section{Practical Evaluation of the Vegetation Interaction Game}

The subjects were 17 sixth grade children (12 years old) in a Japanese elementary school. We recruited 18 children to participate in the experiment, and the participants were divided into three groups $(6,6$, and 5 ) in random order (one child was absent on the experiment day). Game play was held in the elementary school (Fig. 2). Children played the game three times; in each game, the numbers of each event card were controlled so that children could experience some patterns of vegetation succession. After playing the games, the children had a fieldwork activity in Mt. Rokko area experiencing real vegetation succession along with the guidance of

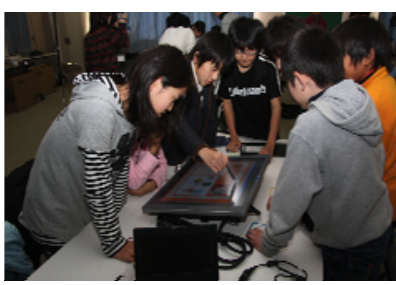

Fig. 2. Game Playing

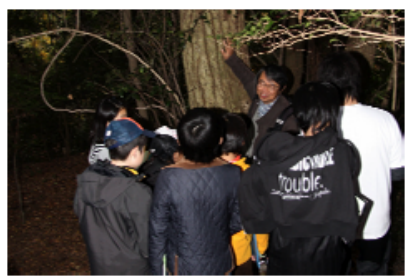

Fig. 3. Field work activity phytosociologist who is proficient at Mt. Rokko area (Fig. 3). 


\subsection{Evaluation of Students' Interests}

After the game play, children answered the questionnaire about the game. Table 1 shows the question items and their results. To examine the children's answers, "think so" and "quite think so" were evaluated as a positive answer, "no opinion," "don't quite think so" and "don't think so" were evaluated as a negative answer. After that, Fisher's exact test was conducted. The results show that positive answers were significantly higher than negative ones in all the items.

Table 1. Results of students' interests

\begin{tabular}{|l|l|l|l|l|l|}
\hline Questions & TS & QTS & NO & DQTS & DTS \\
\hline 1) I enjoyed playing the game.** & 13 & 4 & 0 & 0 & 0 \\
\hline 2) I was absorbed in playing the game. ${ }^{* *}$ & 15 & 2 & 0 & 0 & 0 \\
\hline $\begin{array}{l}\text { 3) I understand the relation between disturbances and the } \\
\text { number of the kinds of plants. }\end{array}$ & 10 & 5 & 0 & 2 & 0 \\
\hline $\begin{array}{l}\text { 4) I understand the characteristics of interactions in } \\
\text { vegetation succession.** }\end{array}$ & 10 & 7 & 0 & 0 & 0 \\
\hline
\end{tabular}

TS; Think so, QTS; Quite think so, NO; No opinion, DQTS; Don't quite think so, DTS; Don't think so, ${ }^{* *} p<0.1$

\section{Conclusion}

In this study, we redesigned and developed a Vegetation Interaction Game and conducted a practical evaluation that include fieldwork activity. The results of evaluation showed that children who participated in the experiment felt the game was interesting and they also thought that they understood the mechanisms of vegetation succession. It was shown that the game could successfully communicate with students, and was able to support their understanding of the relationship between disturbance and the numbers of each plant.

\section{Acknowledgement}

This research is supported by Grants-in-Aid for Scientific Research (B) for Masanori Sugimoto, and Hayao Nakayama Foundation for Science \& Technology and Culture for Akiko Deguchi.

\section{References}

1. Squire, K., Klopfer, E.: Augmented reality simulations on handheld computers. The Journal of the Learning Sciences 16(3), 371-413 (2007)

2. Deguchi, A., Inagaki, S., Kusunoki, F., Yamaguchi, E., Takeda, Y., Sugimoto, M.: Development and Evaluation of a Digital Vegetation Interaction Game for Children. In: Natkin, S., Dupire, J. (eds.) Entertainment Computing - ICEC 2009. LNCS, vol. 5709, pp. 288-289. Springer, Heidelberg (2009) 